\title{
Factors Associated with Psychological Well-Being and Stress: A Cross-Cultural Perspective on Psychological Well-Being and Gender Differences in a Population of Teachers
}

\author{
Ioannis Katsantonis ${ }^{1 \star}$
}

${ }^{1}$ Department of Primary Education, University of Patras, GREECE

*Corresponding Author: ioanniskatsantonis@upnet.gr

Citation: Katsantonis, I. (2020). Factors Associated with Psychological Well-Being and Stress: A Cross-Cultural Perspective on Psychological WellBeing and Gender Differences in a Population of Teachers. Pedagogical Research, 5(4), em0066. https://doi.org/10.29333/pr/8235

\begin{abstract}
ARTICLE INFO
Received: 8 Apr. 2020

Accepted: 27 Apr. 2020

ABSTRACT

The goal of the present study is to examine mainly the associations of contextual variables with stress and teachers' psychological well-being across organizational cultures. The responses $(N=51,782)$ of a population of primary school teachers from 15 different educational cultures were analyzed. Structural equation modeling (SEM) was implemented to confirm the associations of protective factors with stress and workplace well-being at the individual unit of analysis. Following the individual-level analysis, multiple-group SEM (MGSEM) was conducted to examine whether these relations are equivalent across gender groups across cultures. Next, a series of multiple-group confirmatory factor analyses (MGCFA) were carried out to compare the factor intercepts of the dimensions of work-related stress and workplace well-being within clusters of similar organizational cultures. Results indicated that only a limited number of structural associations vary as a function of gender across all cultures at the individual unit of analysis. Mixed results were found in favor of within-cluster homogeneity of latent means. That is, some cultural clusters such as the East-Asian were displaying within-cluster heterogeneity with large latent differences in workload stress. These findings have implications for the development of mental and emotional disorders such as depression or burnout as well as for the prevention of these conditions within educational settings.
\end{abstract}

Keywords: psychological well-being, stress, protective factors, gender-related individual differences, multiplegroup structural equation modeling (MGSEM), multiple-group confirmatory factor analysis (MGCFA), educational cultures, cross-cultural research, measurement invariance, structural invariance

\section{INTRODUCTION}

Admittedly, the teaching profession has been included among the most stressful professional careers (De Simone, Cicotto, \& Lampis, 2016; Kamtsios, 2018; Katsantonis, in-press; Mearns \& Cain, 2003). Stress may negatively affect mental (Bong \& Riding, 1991; Schonfeld, Bianchi, \& Luehring-Jones, 2017) and physical (Bong \& Riding, 1991) health and specifically it may be the cause of the manifestation of burnout, depressive syndromes, psychosomatic symptoms and other mental disorders such as major depression (American Psychiatric Association, 2013). Job-related stress is specifically defined as a psychosomatic and affective reaction to the absence of equilibrium among demanding conditions in the job, the availability of resources, and the subject's existing capacities. Generally, job-related stress, as applied to the educational context, has a multitude of causes that are differently influenced by specific cultures (Klassen et al., 2013).

To date, many empirical studies have identified factors that influence job-related stress and the psychological well-being of teachers. Among the most prevalent sources of job-related stress are (a) stress that arises from student misbehavior and (b) stress that is caused by heavy workload (Collie, Shapka, \& Perry, 2012). From the research perspective of the protective factors, the international literature suggests that variables such as self-efficacy (cf., Katsantonis, in-press; Klassen et al., 2013; Schwarzer \& Hallum, 2008), contextual factors such as the organizational climate (Collie et al., 2012; Katsantonis, 2020), job satisfaction (Ainley \& Carstens, 2018; Katsantonis, 2020), and team innovativeness (Ainley \& Carstens, 2018) play an important role in fostering psychological well-being and reducing work-related stress.

Another interesting line of research in the field of job-related stress in nonclinical populations is the gender-related individual differences in stress. Psychiatric research has shown that males' and females' psychological and physiological responses to stress are differentiated (Verma, Balhara, \& Gupta, 2011). These gender-related individual differences are prevalent in the majority of 
stress-induced mental disorders recorded in the Diagnostic and Statistical Manual of Psychiatric Disorders (American Psychiatric Association, 2013) and have been suggested as determinants of gender-related threats to health (Verma et al., 2011).

As can be seen, despite the breadth of the existing literature on job-related stress, as applied to the educators' context, there is much to be learned from cross-cultural research perspectives both on gender-related differences and on stress levels. Therefore, this study seeks to point out the necessity of investigating these phenomena under culturally diverse settings with primary school teachers in order to prevent mental health problems.

\section{Theoretical Framework}

Self-efficacy refers to the self-evaluation of a subject's capacity to become an agent in a specific domain (e.g., teaching) by initiating the transformation of knowledge and capabilities into actions (Bandura, 1997). Recent empirical findings have shown that self-efficacy can play a proactive role against teachers' work-related stress and have a facilitating role in helping teachers with coping with job-related stress. For example, the study by Katsantonis (in-press) has shown using a sample of primary school teachers that self-efficacy is an important variable in coping with job-related stress. Another study in the Chinese context has also underscored the fact that self-efficacy and support in the form of social climate are the most important factors in coping with stress (Shen, 2009). Multiple other studies, that also corroborate with these findings (e.g., Helms-Lorenz \& Maulana, 2015; Schwarzer \& Hallum, 2008; Skaalvik \& Skaalvik, 2017), have shown that perceived self-efficacy reduces stress.

Another line of research has examined the links between job satisfaction and stress. At this point, it should be mentioned that job satisfaction refers loosely to a sense of fulfillment and gratification that arises from a positive perception of the work's environment (see Collie et al., 2012; Katsantonis, 2020). For instance, the majority of empirical studies have found job satisfaction to be negatively associated with dimensions of work-related stress (cf., Collie et al., 2012; Pecino et al., 2019; Skaalvik \& Skaalvik, 2017). The results of the study by Pecino et al. (2019) have not only supported this statement but have also confirmed the negative association of organizational climate and work-related stress. That is, if there is a positive organizational climate, then the participants have lower levels of stress. The organizational climate in the case of the school context translates to school climate. The last construct is too complex to define. Due to space limitations, suffice to say that the school climate is a superordinate concept that subsumes all types of interpersonal interactions in schools, as well as all infrastructures (Katsantonis, 2020). The beneficial effect of school climate on teachers' psychological well-being is well-documented in the international literature. For example, the study by Collie et al. (2012) has shown that school climate reduces workload stress and student behavior stress.

In parallel to the above, innovativeness has also a role to play in teachers' well-being. According to Ainley and Carstens (2018), innovativeness in educational contexts refers (a) to innovative instructional practices, (b) to the adoption of innovative practices by educators in order to foster change in educational systems, and (c) to schools that are supportive of innovation. Empirical research has shown that innovativeness is closely connected to greater well-being (Honkaniemi, Lehtonen, \& Hasu, 2015). Thus, it is reasonable to assume that innovativeness will have a protective effect against work-related stress.

Despite the aforementioned protective factors, of great importance for the present approach are the cross-cultural differences in work-related stress. Although a few studies (cf., Jamal, 1999; Klassen et al., 2013) have examined the way in which work-related stress is functioning across diverse cultural groups of teacher participants, most of these studies were conducted with a limited number of cultural groups and with different variables as these presented here. Specifically, the study by Klassen et al. (2013) examined how self-efficacy decreased the effects of workload and student behavior stress on job commitment with teacher participants from Canada, England, Hong Kong, and Thailand. On the other hand, the study by Jamal (1999) investigated in Pakistan and Canada bivariate associations of job stress with well-being, which was operationalized as burnout, motivation, job engagement, and attrition.

In addition to the presented literature, another field of inquiry has focused on the gender-related individual differences in stress and response to stress. Admittedly, female teacher participants in various empirical studies are predominantly vulnerable/ more sensitive to stress as compared to their male coworkers. Specifically, the research conducted by Boström et al. (2020) drew a sample of Swedish primary school teachers and reached the conclusion that female participants perceived increased levels of job-related stress, higher levels of emotional demands, and unsatisfying psychosocial environment conditions. Further, Matud, López-Curbelo, and Fortes (2019) underscore the fact that the pertinent studies of gender-related individual differences in psychological well-being processes do not concede. Moreover, these authors showed that gender does, indeed, play a major role in differentiating their participants' responses in psychological well-being.

From the cross-cultural perspective, the findings of the Australian study by Timms, Graham, and Caltabiano (2006) corroborated with these of Boström et al. (2020), that is, their female teacher participants scored higher in stress and burnout measures than their male counterparts. Similar evidence has also been reported regarding the general population in other singlecountry studies (e.g., Matud, 2004) and in cross-country studies (e.g., Miller et al., 2004). Of interest is the study by Miller et al. (2004), which contradicts the commonly thought of gender-related differences, and shows that there are no gender-related individual differences in the sources of stress across cultures.

\section{The Present Study}

The investigation of possible protective factors against work-related stress across educational cultures and genders is important to reduce the future manifestation of mental and emotional disorders in nonclinical populations of educators. Although a few cross-cultural studies that have attempted to identify the mechanisms of stress in nonclinical populations of teachers exist (cf., Jamal, 1999; Klassen et al., 2013), these studies have not considered the possible effect of gender on the relations among the constructs under study. Further, their scope is more limited than that of the proposed study because they included fewer diverse cultural settings. This study is among the first ones, to the best of our knowledge, to examine whether the correlations among 
Table 1. Distribution of the sample across countries and genders

\begin{tabular}{|c|c|c|c|}
\hline Country & Female & Male & Total \\
\hline Argentina & 2156 & 358 & 2514 \\
\hline United Arab Emirates & 8041 & 1147 & 9188 \\
\hline Australia & 2601 & 429 & 3030 \\
\hline Flemish Community Belgium & 2193 & 469 & 2662 \\
\hline Denmark & 1947 & 645 & 2592 \\
\hline England & 1661 & 348 & 2009 \\
\hline Spain & 5536 & 1710 & 7246 \\
\hline France & 1247 & 182 & 1429 \\
\hline Japan & 2038 & 1270 & 3308 \\
\hline Korea & 2518 & 689 & 3207 \\
\hline Netherlands & 1274 & 230 & 1504 \\
\hline Sweden & 1971 & 433 & 2404 \\
\hline Turkey & 2074 & 1130 & 3204 \\
\hline Chinese Taipei & 2535 & 959 & 3494 \\
\hline Viet Nam & 3173 & 818 & 3991 \\
\hline Total & 40965 & 10817 & 51782 \\
\hline
\end{tabular}

Table 2. Item wordings of the workplace well-being and stress scale

1. I experience stress in my work

2. My job leaves me time for my personal life

3. My job negatively impacts my mental health

4. My job negatively impacts my physical health

Note: Reverse-scoring was applied as necessary. Item wordings were retrieved from OECD (2019)

protective factors and psychological well-being are being differentiated across gender groups in a cross-cultural sample of teachers. Of great notice is also the fact that the prior research literature did not jointly consider variables such as team innovativeness and school climate (cf., Katsantonis, in-press; Shen, 2009). Finally, this study aims to provide a cross-cultural perspective on the processes of psychological well-being and job-related stress within specific clusters of structurally homogenous educational cultures by proxy of the countries. Therefore, the subsequent hypotheses were formulated:

$\mathrm{H} 1$ : The dimensions of self-efficacy, job satisfaction, school climate, and team innovativeness will act as protective factors against the dimensions of job-related stress at the individual unit of analysis.

$\mathrm{H} 2$ : The associations among protective factors, well-being, and stress will vary as a function of gender.

H3: No significant differentiation would exist in the levels of psychological well-being and stress in the clusters of approximately homogenous educational cultures.

\section{METHOD}

\section{Participants}

The data of this study were drawn from the Teaching and Learning (TALIS) large-scale international survey of the Organization of Economic Cooperation and Development (OECD). The sample of the current study comprised N=51,782 primary school teachers across fifteen countries. It is noted that OECD surveyed the primary school teachers from late 2017 to early 2018. The data were finalized and published in-detail in March 2020. The present study's sample comprised $N=40,965$ female teachers and $N=10,817$ male teachers. OECD obtained ethical clearance before conducting the international survey and all countries and participants consented to participate. Detailed information on the distribution of the sample across countries is presented in Table 1.

\section{Measures}

\section{Workplace well-being and stress scale}

This 4 items scale was designed by OECD (2019) to measure how the workplace conditions of the teaching profession impact life outside the working environment. All items were given on a 4-point scale ranging from 1 "not at all" to 4 "A lot". McDonald's (1999) omega coefficient of reliability ranges from 0.819 to 0.920 for all 15 countries. Higher scores on this scale are interpreted as lower levels of workplace well-being. Only configural invariance was established across the 15 countries. Item wordings are presented in Table 2.

\section{Workload stress scale}

This 5 items scale was designed by OECD (2019) to measure factors that cause workload stress to the participants. All items were given on a 4-point Likert-type scale ranging from 1 "not at all" to 4 "A lot". McDonald's (1999) omega coefficient of reliability ranges from 0.696 to 0.859 for all 15 countries. Higher scores on this scale are interpreted as higher levels of workload stress. Configural and metric invariance were established across the 15 countries. Item wordings are presented in Table 3. 
Table 3. Item wordings of the workload stress scale

1. Having too much lesson preparation

2. Having too many lessons to teach

3. Having too much marking

4. Having too much administrative work to do (e.g. filling out forms)

5. Having extra duties due to absent teachers

Note: Item wordings were retrieved from OECD (2019)

Table 4. Item wordings of the student behavior stress scale

1. Being held responsible for students' achievement

2. Maintaining classroom discipline

3. Being intimidated or verbally abused by students

Note: Item wordings were retrieved from OECD (2019)

Table 5. Item wordings per school climate subscale

\section{Teachers' perceived disciplinary climate}

1. When the lesson begins, I have to wait quite a long time for students to quieten down

2. Students in this class take care to create a pleasant learning atmosphere

3. I lose quite a lot of time because of students interrupting the lesson

4. There is much disruptive noise in this classroom

\section{Teacher-student relations}

1. Teachers and students usually get on well with each other

2. Most teachers believe that the students' well-being is important

3. Most teachers are interested in what students have to say

4. If a student needs extra assistance, the school provides it

Participation among stakeholders

1. This school provides staff with opportunities to actively participate in school decisions

2. This school provides parents or guardians with opportunities to actively participate in school decisions

3. This school provides students with opportunities to actively participate in school decisions

4. This school has a culture of shared responsibility for school issues

5. There is a collaborative school culture which is characterised by mutual support

Note: Reverse-scoring was applied as necessary. Item wordings were retrieved from OECD (2019)

\section{Student behavior stress scale}

This 3 items scale was also designed by OECD (2019) to measure the causes of stress that arise from the interaction with students. Specifically, this scale pertains to stress caused by students' behavior. McDonald's (1999) omega coefficient of reliability ranges from 0.669 to 0.978 for all 15 countries. Higher scores on this scale are interpreted as higher levels of stress that are caused by students' behavior. Configural and metric invariance were established across the 15 countries. Item wordings are presented in Table 4.

\section{School climate scales}

Three scales represent various dimensions of organizational climate as applied to the school context, namely teachers' perceived disciplinary climate, teacher-student relations, and participation among stakeholders. All scales were created and administered by OECD (2019). All items for each scale were given on a 4-point Likert-type scale ranging from 1 "Strongly disagree" to 4 "Strongly agree". The scale of "teachers' perceived disciplinary climate" comprises 4 items that describe the participants' perceptions of issues of student (mis-) behavior. Higher scores on this scale reflect greater levels of student misbehavior. McDonald's (1999) coefficient omega ranged from 0.780 to 0.914 across all countries. Configural and metric invariance were established across the 15 countries. The second 4 items scale pertains to the "teacher-student relations". This scale is composed of items that refer to teacher-student interactions. Higher scores on this scale reflect greater levels of positive relations among teachers and students. McDonald's (1999) coefficient omega ranged from 0.771 to 0.914 across all countries. Configural and metric invariance were established across the 15 countries. Finally, the "participation among stakeholders" scale comprised 5 items that referred to the decision making in schools. Higher scores on this scale reflect greater levels of participation of all involved parties in decision making. McDonald's (1999) coefficient omega ranged from 0.729 to 0.912 across all countries. Configural and metric invariance were established across the 15 countries. Item wordings per school climate subscale are presented in Table 5.

\section{Self-efficacy scales}

Self-efficacy is domain-specific and three scales reflect the self-efficacy of teachers. All scales were administered by OECD (2019). All items were given on a 4-point Likert-type scaling scheme ranging from 1 "Not at all" to 4 "A lot". These self-efficacy scales were an adaption of the widely used teacher self-efficacy scale (see Katsantonis, 2020). The first scale, namely "self-efficacy in classroom management", reflects the participants' self-referent beliefs of capabilities in managing student behavior. Higher scores in this scale reflect higher levels of self-capacity to control students' behavior in the classroom. McDonald's (1999) coefficient omega ranged from 0.757 to 0.904 across all countries. Configural and metric invariance were established across the 
Table 6. Item wordings per self-efficacy subscale

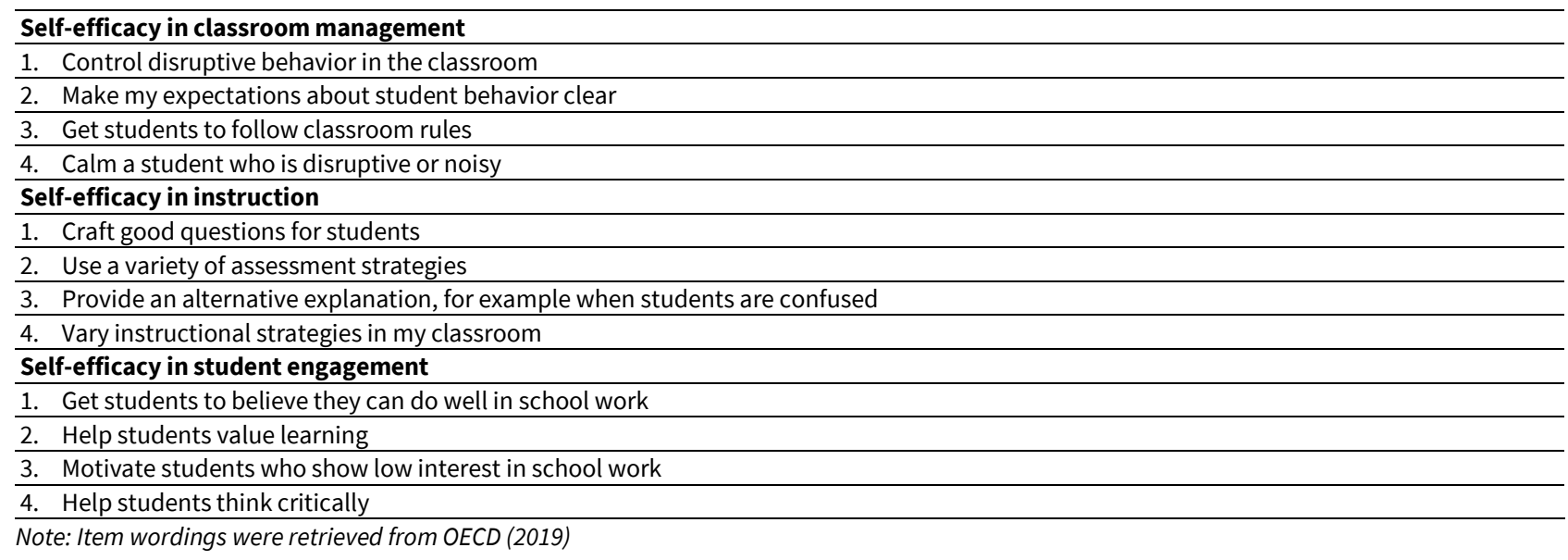

Table 7. Item wordings per job satisfaction scale

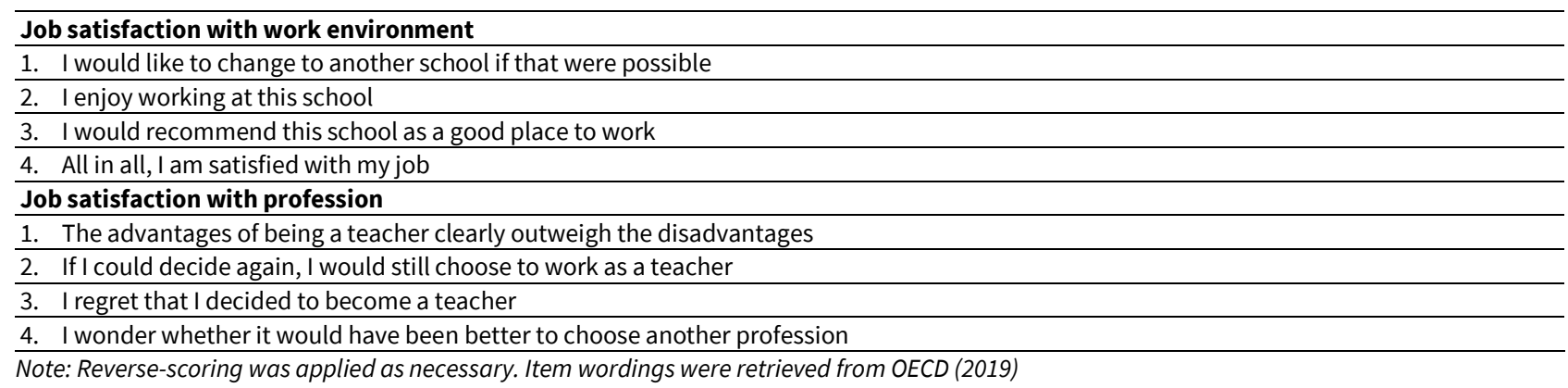

Table 8. Item wordings of team innovativeness scale

\begin{tabular}{ll}
\hline 1. & Most teachers in this school strive to develop new ideas for teaching and learning \\
\hline 2. & Most teachers in this school are open to change \\
\hline 3. & Most teachers in this school search for new ways to solve problems \\
\hline 4. & Most teachers in this school provide practical support to each other for the application of new ideas \\
\hline Note: Item wordings were retrieved from OECD (2019)
\end{tabular}

15 countries. The second scale, namely "self-efficacy in instruction", reflects the participants' self-assessment of their capacity to implement instructional strategies. Higher scores on this scale reflect higher levels of self-capacity to implement adaptive instructional strategies. McDonald's (1999) coefficient omega ranged from 0.719 to 0.874 across all countries. Configural and metric invariance were established across the 15 countries. Finally, the third scale, namely "self-efficacy in student engagement", refers to the participants' capacity to engage students in the classroom setting. Higher scores on this scale reflect higher levels of self-capacity to engage students. McDonald's (1999) coefficient omega ranged from 0.704 to 0.821 across all countries. Configural and metric invariance were established across the 15 countries. Item wordings per subscale are presented in Table 6.

\section{Job satisfaction scales}

Two scales reflect two dimensions of job satisfaction, namely "job satisfaction with the environment" and "job satisfaction with profession." All scales were created and administered by OECD (2019). All items were scored using a 4-point Likert-type scaling scheme ranging from 1 "Strongly disagree" to 4 "Strongly agree." Higher scores on this scale reflect higher levels of satisfaction with the working environment. McDonald's (1999) coefficient omega ranged from 0.767 to 0.874 across all countries. Configural and metric invariance were established across the 15 countries. The second scale comprised four items that reflect satisfaction with the profession. Higher scores on this scale reflect higher levels of satisfaction with the profession. McDonald's (1999) coefficient omega ranged from 0.702 to 0.947 across all countries. Configural and metric invariance were established across the 15 countries. Item wordings are presented in Table 7.

\section{Team innovativeness scale}

Because team innovativeness has been proven as an important factor in psychological well-being, prevention of burnout, and attrition (Ainley \& Carstens, 2018), it was included in the subsequent analyses. Team innovativeness is composed of 4 items of general innovativeness. All items were scored on a 4-point Likert-type scale ranging from 1 "Strongly disagree" to 4 "Strongly agree." Higher scores on this scale reflect greater general innovativeness. McDonald's (1999) coefficient omega ranged from 0.931 to 0.958 across all countries. Configural, metric, and scalar invariance was established across the 15 countries. Item wordings are presented in Table 8. 
Table 9. Latent Correlation Matrix

\begin{tabular}{|c|c|c|c|c|c|c|c|c|c|c|c|c|}
\hline & 1 & 2 & 3 & 4 & 5 & 6 & 7 & 8 & 9 & 10 & 11 & 12 \\
\hline 1. Clman & 1 & 0.809 & 0.861 & 0.219 & 0.190 & -0.252 & 0.224 & 0.125 & -0.045 & -0.224 & -0.121 & 0.105 \\
\hline 2.. Inst & & 1 & 0.918 & 0.224 & 0.207 & -0.112 & 0.247 & 0.176 & -0.064 & -0.160 & -0.116 & 0.158 \\
\hline 3. Steng & & & 1 & 0.235 & 0.235 & -0.157 & 0.206 & 0.173 & -0.090 & -0.186 & -0.168 & 0.158 \\
\hline 4. Env & & & & 1 & 0.589 & -0.196 & 0.461 & 0.551 & -0.198 & -0.282 & -0.478 & 0.354 \\
\hline 5. Prof & & & & & 1 & -0.168 & 0.254 & 0.245 & -0.309 & -0.318 & -0.523 & 0.157 \\
\hline 6. Disp & & & & & & 1 & -0.139 & -0.140 & 0.170 & 0.524 & 0.284 & -0.094 \\
\hline 7. Rel & & & & & & & 1 & 0.522 & -0.065 & -0.159 & -0.141 & 0.410 \\
\hline 8. Рcip & & & & & & & & 1 & -0.178 & -0.167 & -0.272 & 0.475 \\
\hline 9. Load & & & & & & & & & 1 & 0.598 & 0.552 & -0.088 \\
\hline 10. Beh & & & & & & & & & & 1 & 0.528 & -0.057 \\
\hline 11. Wstr & & & & & & & & & & & 1 & -0.181 \\
\hline 12.. Innov & & & & & & & & & & & & 1 \\
\hline
\end{tabular}

Note: All latent, error-free correlations are significant at p<0.001. Clman: Classroom management self-efficacy; Inst: Instruction self-efficacy; Steng: Student engagement self-efficacy; Env: Job satisfaction with the environment; Prof: Job satisfaction with the profession; Disp: Teachers' perceived disciplinary climate; Rel: Teacher-student relations; Pcip: Participation among stakeholders; Load: Workload stress; Beh: Student behavior stress; Wstr: Workplace well-being and stress; Innov: Team innovativeness.

\section{Procedure}

In the present study, all structural and measurement parameters inside the overarching framework of structural equation modeling (SEM) were estimated with the Robust Maximum Likelihood (MLR) estimator with the Yuan- Bentler correction in the Lavaan package (Rosseel, 2012) in the statistical language and environment R (R Core Team, 2018). The MLR estimator is known to perform considerably well with data with 4 categories (cf., Bandalos, 2014; Li, 2016; Rhemtulla, Brosseau-Liard, \& Savalei, 2012). Due to computational demands, listwise deletion of missing cases was automatically implemented for all analyses. The latent variables were scaled by standardizing the factor variance to unity, following Brown's (2015) recommendation.

All cross-cultural analyses were not conducted using the full sample of 15 countries because of great cross-group heterogeneity (Davidov et al., 2014). Instead of utilizing all countries, we adopted the taxonomy of Bulle (2011) and formed clusters of similar educational cultures. That is, according to Bulle (2011), Japan and Korea form the East- Asian model. France and Spain form the Latin model. Denmark and Sweden form the Northern model. Australia and the United Kingdom represent the AngloSaxon model and finally, Belgium and the Netherlands form the Germanic model. We restricted our further analyses to these clusters of similar educational cultures because it is assumed that within-cluster, the educational systems would impose similar demands on their respective populations and would also display greater similarity regarding their structural beliefs. Clustering of countries based on similar characteristics is quite common within the cross-cultural and cross-national research designs (Byrne \& van de Vijver, 2010; Davidov et al., 2014).

\section{RESULTS}

It is noted that in the present study values equal to or greater than 0.90 for CFI and TLI are indicative of acceptable model-data fit. RMSEA and SRMR values less than 0.08 are also considered acceptable, but preferably values less than 0.05 are desirable (see Hu \& Bentler, 1999). Specially designed sampling weights were included in all pooled-sample, individual-level analyses to correct the unequal selection probabilities of the sampling units as well as to ensure equal representativeness of all countries in goodnessof-fit indices in SEM analyses (OECD, 2019).

At the beginning of the analyses, the full sample of $\mathrm{N}=51,782$ participants was utilized to calculate a latent correlation matrix, that describes the individual latent associations among the theoretical constructs. The matrix is presented in Table 9. The goodness-of-fit indices displayed the following values: $\mathrm{X}^{2}(\mathrm{df}=1055, \mathrm{~N}=37,541)=21213.779, p<0.001, \mathrm{CFI}=0.932, \mathrm{TLI}=0.924, \mathrm{RMSEA}=$ $0.04190 \% \mathrm{Cl}[0.040 ; 0.041]$, SRMR $=0.044$. It is noted that the standardized factor loadings $(\lambda \mathrm{s})$ across all factors ranged from 0.167 (one item) to 0.899 , which shows that the majority of items had great psychometric properties (Brown, 2015). It is underscored that only one item had a factor loading below the 0.35 rule, but it was retained in the analyses, as was done also by OECD (2019). The path diagram of the model is presented in Figure 1. 


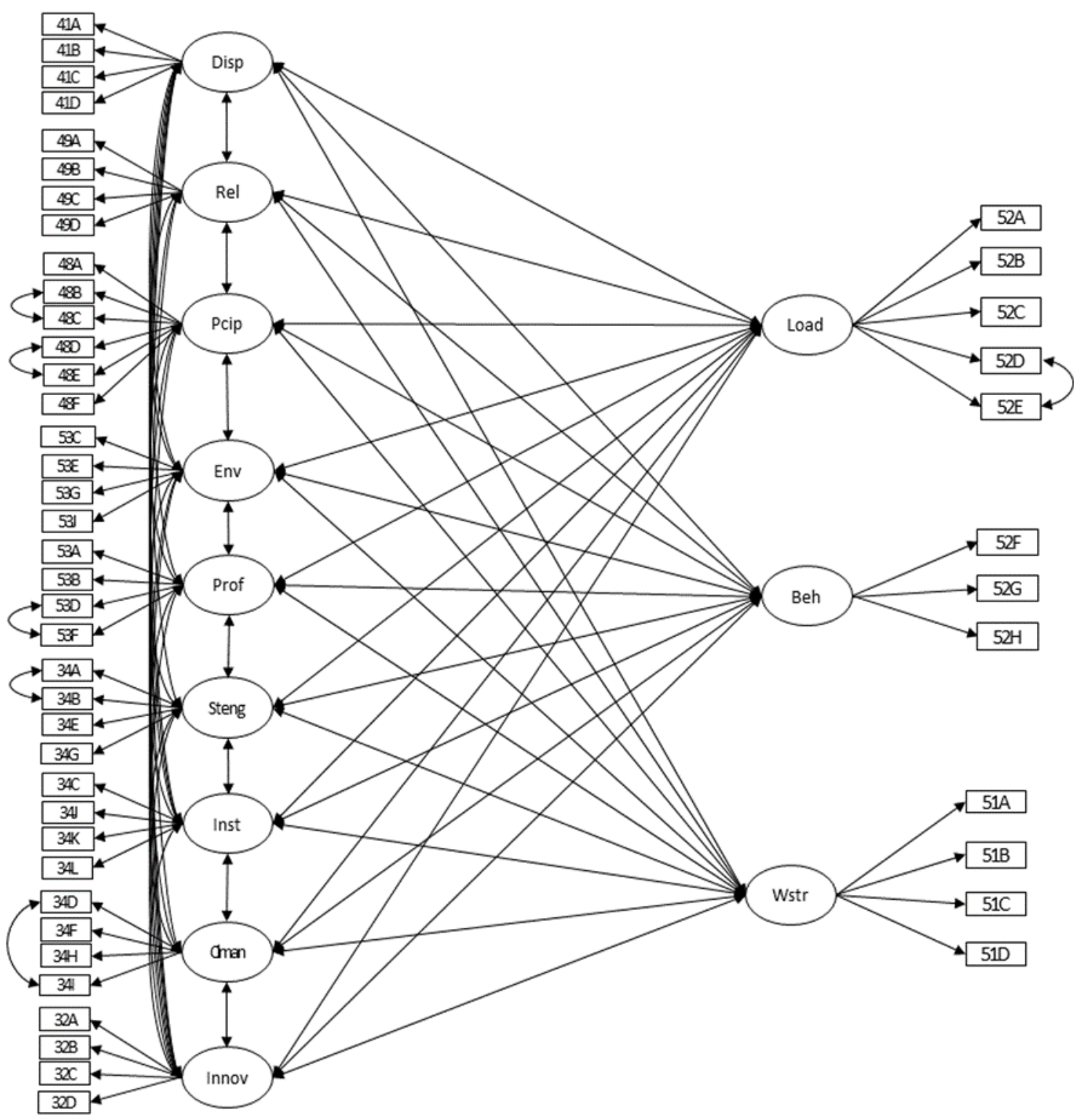

Figure 1. Path diagram- "Interfactor Covariances Among Protective Factors and Workplace Well-being, Workload Stress, and Student Behavior Stress"- All lines among factors are bidirectional arrows.

As the research objective is the investigation of whether the structural relations among protective factors, work-related stress, and well-being vary as a function of gender, we followed up our analyses with multiple-group SEM (MGSEM) in order to examine structural invariance of interfactor covariances.

Testing the equivalence of the interfactor covariances across groups is a higher level of invariance that is preceded by measurement invariance. Measurement invariance is established if $|\triangle C F I| \leq 0.010$ and $|\triangle R M S E A| \leq 0.015$ (Cheung \& Rensvold, 2003; Chen, 2007). However, according to Chen (2007), the main criterion is always the $|\Delta C F I| \leq 0.010$ because it is not influenced by model complexity and sampling adequacy. In the case of non-invariance, a partially invariant model (Byrne, Shavelson, \& Muthen, 1989; Davidov et al., 2014) would be considered following (a) the Lagrange Multiplier (LM) tests (Bentler, 2006) with the largest chi-square value and (b) the degree of variability of the non-invariant parameters until the $\Delta$ approximate goodness-of-fit indices $(\Delta \mathrm{AFI})$ fall within the acceptable limits. The only assumption for this procedure is that at least two items' factor loadings and their associated intercepts are equal across groups (see Brown, 2015; Steenkamp \& Baumgartner, 1998). A case of metric non-invariance would mean that the non-invariant items were cognitively construed differently within-cluster and the psychological meaning would not be the same across similar educational cultures. The $\Delta$ approximate goodness-of-fit $(\Delta \mathrm{AFI})$ indices of the progressively more constrained models are presented in Table 10. It is noted that only the equivalence of the psychological meaning (i.e., metric invariance) is necessary before proceeding with structural invariance testing (Bentler, 2006; Byrne, 2016). 
Table 10. Measurement and Structural Invariance Across Gender Groups

\begin{tabular}{|c|c|c|c|c|c|}
\hline Level of Invariance & CFI & $|\Delta C F I|$ & RMSEA & |DRMSEA| & $\begin{array}{c}\text { Satorra- Bentler } \\
\mathrm{X}^{2}(\mathbf{d f})\end{array}$ \\
\hline \multicolumn{6}{|c|}{ Measurement Invariance } \\
\hline Configural & 0.931 & & 0.041 & & $22630.568^{\star \star \star}(2110)$ \\
\hline Metric $\dagger$ & 0.931 & 0.000 & 0.041 & 0.000 & $22744.283^{\star \star \star}(2147)$ \\
\hline \multicolumn{6}{|c|}{ Structural Invariance } \\
\hline Equal Latent Covariances & 0.931 & 0.000 & 0.041 & 0.000 & $22853.202^{\star \star \star}(2213)$ \\
\hline Partial Latent Covariances & 0.931 & 0.000 & 0.041 & 0.000 & $22770.800^{\star \star \star}(2199)$ \\
\hline
\end{tabular}

Note: $†$ Freely estimated covariances but equal loadings. ${ }^{* *}$ Significant at $p<0.001$.

We also rely on the Satorra- Bentler (2001) delta chi-square test of differences between nested models to identify localized areas of strain (Sass \& Schmitt, 2013). The Satorra- Bentler scaling corrected $\Delta X^{2}(66)=136.29, p<0.001$, indicated that the latent covariances were not invariant across gender groups as compared to the metric model with freely estimated structural parameters. Suffice to say that the Lagrange Multiplier tests indicated that 11 latent covariances and 3 loadings of the self-efficacy scales were non-invariant across gender groups. Among these non-invariant covariances, those most pertinent to our study are between satisfaction with profession and workload stress $\left(r_{\text {males }}=-0.286, p<0.001 ; r_{\text {females }}=-0.329, p<0.001\right)$; between teacherstudent relations and workload stress $\left(r_{\text {males }}=-0.153, p<0.001 ; r_{\text {females }}=-0.046, p<0.001\right)$; between participation among shareholders and workplace well-being ( $\left.r_{\text {males }}=-0.264, p<0.001 ; r_{\text {females }}=-0.275, p<0.001\right)$. By relaxing these equality constraints, the SatorraBentler $\Delta x^{2}(52)=43.514, p>0.05$, indicated that the partially invariant, latent covariances model as compared to the metric model was not degraded from a model fit perspective.

To compare latent means in dimensions of work-related stress and workplace well-being across the different cultural settings, the clusters of countries will be utilized. Measurement equivalence in cross-national research is typically investigated via multiplegroup confirmatory factor analyses (MGCFA- Davidov et al., 2014). Specifically, in this study, a series of unidimensional (i.e., onefactor) models would be fitted in each cluster separately and measurement invariance will be sought across three levels of progressively more constrained models, i.e., configural, metric, and scalar models. It is noted that this approach is by far more superior because it takes into consideration both the latent nature of the dimensions of stress and well-being, as well as the measurement error that is inherent in self-report measures (see Brown, 2015). The "student behavior stress" factor's invariance would not be examined because the model would be just-identified and thus no fit indices could be calculated (Kline, 2016).

First, the Anglo-Saxon cluster was examined. The approximate fit indices indicated that the "workplace well-being and stress" factor was metric invariant in the Anglo-Saxon cluster with $|\triangle C F I|=0.003$ and $|\triangle R M S E A|=0.022$. The $|\Delta C F I|$ was equal to 0.009 and the $|\triangle \mathrm{RMSEA}|=0.002$ for the scalar model. The equal factor intercepts model was invariant as compared to the scalar model, $|\Delta \mathrm{CFI}|=$ 0.002 and $|\triangle R M S E A|=0.002$. In the same cluster, the configural model for the "workload stress" factor was exceptionally well-fitting, i.e., $\mathrm{CFI}=0.984$, RMSEA $=0.072$. The metric model as compared to the configural model was invariant, $|\triangle C F I|=0.003$ and $|\triangle R M S E A|=0.010$. The scalar model as compared to the metric one was not invariant, $|\triangle C F I|=0.053$ and $|\triangle R M S E A|=0.040$. The univariate Lagrange Multiplier (LM) tests indicated that the intercepts of items 2 ( $\tau 2$ ), 3 ( $\tau 3$ ), and 5 ( $\tau 5)$ were non-invariant, i.e., $\operatorname{LMX}^{2}{ }_{2}(1)=143.083, p<0.001 ; \operatorname{LMX}^{2}{ }_{3}(1)=178.077, p<0.001 ; \mathrm{LMX}^{2}{ }_{5}(1)=30.184, p<0.001$, respectively. By relaxing these constraints, the $\triangle$ approximate fit indices fell within the acceptable range, $|\triangle C F I|=0.004$ and $|\triangle R M S E A|=0.003$. The factor means were of equal magnitude, $|\triangle \mathrm{CFI}|=0.006$ and $|\triangle \mathrm{RMSEA}|=0.005$.

Regarding the East-Asian cluster, the procedure followed as abovementioned. In the East-Asian cluster, the "workplace wellbeing and stress" factor displayed acceptable metric invariance, i.e., $|\Delta C F I|=0.007$ and $|\Delta R M S E A|=0.007$. Despite the above, the scalar level of invariance was not achieved, $|\triangle C F I|=0.024$ and $|\triangle R M S E A|=0.031$. A partially invariant scalar model was examined following the univariate Lagrange Multiplier (LM) tests. The LM tests indicated that the intercepts of items 1 ( $\tau 1$ ) and 3 ( $\tau 3$ ) were non-invariant, i.e., $\operatorname{LMX}^{2}{ }_{1}(1)=250.454, p<0.001 ; \operatorname{LMX}^{2}{ }_{3}(1)=89.178, p<0.001$. By relaxing the equality constraints on these items, a partially scalar invariant model was achieved, $|\triangle C F|=0.001$ and $|\triangle R M S E A|=0.004$. The factor means were also of equal magnitude within the cluster, $|\triangle C F I|=0.004$ and $|\triangle R M S E A|=0.006$. The "workload stress" reached metric invariance, i.e., $|\Delta C F I|=0.006$ and $|\triangle \mathrm{RMSEA}|=0.008$. However, scalar invariance was not established, $|\triangle C F I|=0.045$ and $|\triangle \mathrm{RMSEA}|=0.034$. The LM tests indicated that the intercepts of items 1 ( $\tau 1), 2$ ( $\tau 2)$, and 3 ( $\tau 3$ ) were non-invariant $\operatorname{LMX}^{2}{ }_{1}(1)=74.034, p<0.001 ; \mathrm{LMX}^{2}{ }_{2}(1)=392.693, p<0.001 ; \mathrm{LMX}{ }^{2}{ }_{3}$ $(1)=81.133, p<0.001$, respectively. By relaxing the equality constraints on these items' intercepts, a partially scalar invariance was established, i.e., $|\triangle C F I|=0.000$ and $|\triangle R M S E A|=0.003$. However, the factor intercepts were not equal, $|\Delta C F I|=0.035$ and $|\triangle R M S E A|=0.037$. To calculate the extend of within-cluster differentiation, Hancock's (2001) extension of Cohen's d, which is called Latent d, was calculated. Japanese participants had greater levels of workload stress than Korean participants. The effect size of that difference was large, i.e., Latent $\mathrm{d}=0.981$.

Next, the Northern cluster is considered. Although metric invariance was established for the "workplace well-being and stress" factor (i.e., $|\triangle C F I|=0.003$ and $|\triangle R M S E A|=0.024)$, scalar invariance was not established, i.e., $|\Delta C F| \mid=0.152$ and $|\triangle R M S E A|=0.123$. The $\mathrm{LM}$ tests indicated that the intercepts of items 2 ( $\tau 2$ ) and 3 ( $(\mathrm{T} 3)$ were non-invariant, $\mathrm{LMX}^{2}{ }_{2}(1)=856.509, p<0.001 ; \mathrm{LMX}^{2}{ }_{1}(1)=67.440$, $p<0.001$, respectively. The partially scalar model compared to the metric model was not degraded, i.e., $|\Delta C F I|=0.001$ and $|\triangle \mathrm{RMSEA}|=0.005$. The factor means were of equal magnitude within-cluster, i.e., $|\triangle C F I|=0.007$ and $|\triangle \mathrm{RMSEA}|=0.006$. Regarding the "workload stress", it should be noted that full metric invariance was not established, $|\triangle C F| \mid=0.031$ and $|\triangle R M S E A|=0.000$. The LM tests indicated that the loadings of items $1(\lambda 1)$ and $3(\lambda 3)$ were non-invariant, i.e., $\operatorname{LMX}^{2}{ }_{1}(1)=99.555, p<0.001 ; \operatorname{LMX}^{2}{ }_{3}(1)=111.280$, $p<0.001$, respectively. By relaxing these constraints, partial metric invariance was established with $|\Delta C F I|=0.008$ and $|\triangle \mathrm{RMSEA}|=0.006$. These items' associated intercepts were left unconstrained at the scalar level; however, scalar invariance was not established, i.e., $|\triangle C F I|=0.090$ and $|\triangle \mathrm{RMSEA}|=0.715$, and the procedure was terminated. 
Following the invariance testing in the Anglo-Saxon, East-Asian, and Northern clusters, the Latin cluster was considered next. Metric invariance was established for the "workplace well-being" factor, i.e., $|\Delta C F I|=0.005$ and $|\triangle R M S E A|=0.023$. Scalar invariance was not established, i.e., $|\triangle C F| \mid=0.082$ and $|\triangle R M S E A|=0.068$, and thus, a partially invariant model was examined. However, the $L M$ tests indicated that three of the four items' intercepts were non-invariant, which violates the assumption of at least two invariant loadings and intercepts. Thus, the procedure was terminated for this factor. Next, the "workload stress" factor was examined. Within-cluster, metric invariance was established, i.e., $|\Delta C F|=0.004$ and $|\triangle R M S E A|=0.017$, however, scalar invariance was impossible to be established because the LM tests indicated that more than 3 intercepts were non-invariant.

Finally, the Germanic cluster was considered for invariance analyses. Although metric invariance was established for the "workplace well-being and stress" factor (i.e., $|\Delta C F I|=0.003$ and $|\triangle R M S E A|=0.022$ ), scalar invariance was not (i.e., $|\Delta C F I|=0.100$ and $|\triangle R M S E A|=0.089)$. Despite the above, a partially invariant scalar model was achieved by relaxing the equality constraints on items' 1 ( $\tau 1)$ and 2 ( $\tau 2)$ intercepts according to the LM tests suggestions, i.e., $\operatorname{LMX}^{2}{ }_{1}(1)=211.624, p<0.001 ; \operatorname{LMX}^{2} 2(1)=327.948, p<0.001$, respectively. The $\Delta$ approximate fit indices fell within the acceptable range (i.e., $|\triangle C F I|=0.000$ and $|\triangle R M S E A|=0.006$ ). The factor intercepts, though, were not equal within-cluster (i.e., $|\triangle C F I|=0.036$ and $|\triangle R M S E A|=0.046$ ). Within the Germanic cluster, participants from Belgium had greater levels of stress as a result of workplace demands. This differentiation was of medium effect size with Latent $\mathrm{d}=0.532$. Regarding the "workload stress" factor, a partially metric model was considered because the full metric model was degraded (i.e., $|\triangle C F|=0.016$ and $|\triangle R M S E A|=0.006)$. The LM test indicated that the loading of item $2(\lambda 2)$ was noninvariant, i.e., $\operatorname{LMX}_{2}{ }_{2}(1)=44.464, p<0.001$. The partially metric model as compared to the configural was invariant (i.e., $|\Delta C F I|=0.002$ and $|\triangle R M S E A|=0.013)$. The associated item intercept was also relaxed in the following scalar model. The scalar model, though, was non-invariant (i.e., $|\triangle C F I|=0.102$ and $|\triangle R M S E A|=0.050)$. The $L M$ tests additionally indicated that the intercepts of items 4 ( $\tau 4)$ and 5

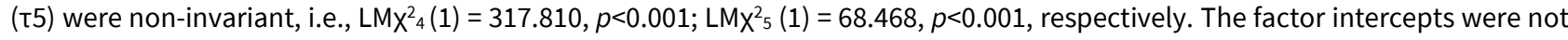
of equal magnitude within-cluster, i.e., $|\Delta C F I|=0.069$ and $|\triangle \mathrm{RMSEA}|=0.017$. That is, participants from Belgium perceived greater workload stress. The effect size was medium with Latent $d=0.692$.

\section{DISCUSSION}

The purpose of the current study was essentially threefold: (a) to examine the associations of various protective factors with the psychological well-being of teachers; (b) to investigate the effect of gender on these relations, and (c) to examine latent mean differences in workplace well-being and stress, and work-related stress across five clusters of approximately, structurally homogenous educational cultures.

Regarding the first research objective, the results indicated that all factors had, indeed, a significant effect. Thus, hypothesis 1 was confirmed. Analytically, the latent correlation matrix (see Table 9) indicated that self-efficacy in instruction was negatively associated with workload stress, student-behavior stress, and workplace well-being and stress. Negative correlation coefficients were also observed for the associations of self-efficacy in classroom management and self-efficacy in student engagement with the three variables that represent psychological well-being in this study. This finding is entirely expected because prior research has stressed the importance of self-efficacy in reducing stress (Helms-Lorenz \& Maulana, 2015; Katsantonis, in-press; Schwarzer \& Hallum, 2008; Skaalvik \& Skaalvik, 2017) and facilitating well-being (Ainley \& Carstens, 2018). Because we believe in a dynamic system between well-being and job satisfaction, we included dimensions of job satisfaction as protective factors. In other words, negative interfactor correlations were found across all dimensions of job satisfaction with well-being and stress; that is, those participants who were more satisfied with their work were also perceiving less stress. This result is also consistent with the findings of other within-country studies (Collie et al., 2012; Pecino et al., 2019; Skaalvik \& Skaalvik, 2017). Of great significance is also the fact that organizational climate, in this instance school climate, is exerting a protective effect against the development of stress. One finding that should be discussed more is the positive correlation coefficient of perceived disciplinary climate with the dimensions of stress. This result shows that school climate may also have a negative effect, that is, it may inflate the levels of workload stress and student-behavior stress. Finally, team innovativeness is also a protective factor, as the literature suggested (see Ainley \& Carstens, 2018; Honkaniemi et al., 2015), but a weak one.

Despite the suggestions of gender-related individual differences in stress and stress responses (e.g., Boström et al., 2020; Matud et al., 2019), this study shows that the latent correlations among the protective factors and the dimensions of psychological well-being are mostly not affected by by gender. In other words, gender does not play a major role in the variability of the relations among the factors that help in reducing work-related stress. This finding could be aligned with the findings of Miller et al. (2000), who found a universal mechanism of the sources of stress at the individual unit of analysis. By transferring this finding to the current study, we could reasonably conclude that that the selected latent associations are not influenced by gender, that is, these relations are universal across gender groups. Thus, hypothesis was partially rejected.

Regarding the third research objective, an unexpected result was observed. That is, both the latent means of workplace wellbeing and workload stress were equivalent within the Anglo- Saxon cluster, but great heterogeneity was observed in the other clusters. Specifically, in the East- Asian cluster, the latent means of the workplace well-being and stress factor were equal in Japan and Korea; however, the latent means of the workload stress factor were unequal. Unequal latent means were also found in the Germanic cluster both for the workplace well-being factor and the workload stress factor. The Latin cluster was not considered for further analyses due to technical issues. In the Northern cluster, the MGCFA showed that the latent means of workplace well-being factor were equal. As can be seen, despite the assumed homogeneity of educational cultures as described in Bulle (2011), medium to large latent differences were found in the levels of psychological well-being of this population of teachers. Thus, hypothesis 3 was rejected. It should be noted that the current study is the first, to the best of our knowledge, to have examined latent differences in psychological well-being and stress of teachers within these five cultural clusters. 


\section{CONCLUSIONS}

In short, a careful inspection of the present findings seems to suggest that personal resources such as self-efficacy and job satisfaction appear to be more powerful protective factors against inflated levels of stress and psychological distress. The current study showed also across various educational cultures that, despite the suggestions of gender-related individual differences in psychological well-being, teachers' gender did not play a major role in the associations between protective factors and psychological well-being and stress. Further, these findings at the individual unit of analysis, at least, demystify some of the underlying mechanisms of gender-related individual differences in coping with job-related stress because they are indicative of the universal role of the pertinent protective resources.

Beyond the matter of individual differences, great heterogeneity in psychological well-being and stress levels was observed in the selected educational clusters, despite the assumed homogeneity of educational cultures in the pertinent clusters. These conclusions can help policy planning in the respective educational systems that show inflated levels of psychological distress. In other words, these results may point toward the necessity of implementing intervention programs in order to reduce stress levels in specific educational cultures. These findings have also implications for the prevention and detection of early symptoms of mental disorders. Stress is known to negatively influence the mental and physiological health of the population (Bong \& Riding, 1991; Schonfeld et al., 2017) and it may induce depression, psychosomatic symptoms, burnout, etc. (American Psychiatric Association, 2013). Therefore, it is necessary to consider protective factors that can help alleviate stress in teachers that are highly susceptible (see De Simone et al., 2016; Kamtsios, 2018; Mearns \& Cain, 2003) to the development of stress and mental disorders. Further, these analyses have made it more apparent that workload stress, student-behavior stress, and workplace well-being are cognitively construed differently even within approximately homogenous cultures. Also, the findings of scalar non-invariance suggest the existence of differential additive response style (Kline, 2016), that is, the participants' responses to the non-invariant items were affected by other factors beyond the latent construct. Moreover, this result shows that even if the participants across cultures had the same standing in the latent traits' continuum, they did not respond by selecting the same options of the scales.

\section{Implications for Future Research}

The current study, according to our opinion, can be considered as the stepping stone for future research in this field in the following ways: The inferences that were supported by this study could be of use in the development of inventories for screening educators for stress-induced psychological conditions. Secondly, the cross-cultural part of this study could also be of help to researchers who investigate stress-related conditions with populations of teachers across diverse educational systems. That is, the interested researchers could try to "unpackage" culture by carefully considering how culture-specific elements in these clusters are responsible for the observed differences in factor intercepts. Further, due to the differential additive response style, that was found in the present study, we strongly recommend the testing of measurement invariance in psychoeducational research that pertains to psychological well-being because it seems that participants do not cognitively construe the meaning of the items in the same way across educational cultures. This could have serious implications for the interpretation and adaption of screening inventories.

\section{Limitations}

Some limitations of the current perspective should be kept in mind while interpreting the results. Firstly, the current study did not consider these associations at the within-culture level, which could have provided rich information regarding the conditions within specific educational cultures that either are among the high- or low-performing in OECD PISA program. Moreover, we did not consider protective factors across educational cultures from a multilevel perspective, which could have helped our understanding of cross-level interfaces. Thus, there is much to be learnt yet by research in this field.

\section{REFERENCES}

Ainley, J., \& Carstens, R. (2018). Teaching and Learning International Survey (TALIS) 2018 Conceptual Framework. Paris, France: OECD Publishing. https://doi.org/10.1787/799337c2-en

American Psychiatric Association. (2013). Diagnostic and Statistical Manual of Mental Disorders (5th eds.). Washington DC, USA: American Psychiatric Association.

Bandalos, D. (2014). Relative performance of categorical diagonally weighted least squares and robust maximum likelihood estimation. Structural Equation Modeling, 21, 102-116. https://doi.org/10.1080/10705511.2014.859510

Bandura, A. (1997). Self-efficacy: The exercise of control. New York, NY, USA: W.H. Freeman and Company.

Bentler, P. (2006). EQS 6 Structural Equations Program Manual. California, CAL, USA: Multivariate Software Inc. Retrieved from https://www3.nd.edu/ kyuan/courses/sem/EQS-Manual6.pdf

Bong, G. M., \& Riding, J.R. (1991). Occupational Stress and Satisfaction in Teaching. British Educational Research Journal, 17, 263281. https://doi.org/10.1080/0141192910170306

Boström, M., Björklund, C., Bergström, G., Nybergh, L., Elinder, S. L., Stigmar, K., ... Kwak, L. (2020). Health and Work Environment among Female and Male Swedish Elementary School Teachers-A Cross-Sectional Study. International Journal of Environmental Research and Public Health, 17, 227. https://doi.org/10.3390/ijerph17010227

Brown, A. T. (2015). Confirmatory factor analysis for applied research (2nd eds.). New York, NY, USA: The Guilford Press. 
Bulle, N. (2011). Comparing OECD educational models through the prism of PISA. Comparative Education, 47, $503-521$. https://doi.org/10.1080/03050068.2011.555117

Byrne, B. (2016). Structural Equation Modeling with AMOS: Basic Concepts, Applications, and Programming (3rd ed.). New York, NY, USA: Routledge.

Byrne, B., \& van de Vijver, J. R. F. (2010). Testing for Measurement and Structural Equivalence in Large-Scale Cross-Cultural Studies: Addressing the Issue of Nonequivalence. International Journal of Testing, 10, $107-132$. https://doi.org/10.1080/15305051003637306

Byrne, B., Shavelson, R., \& Muthen, B. (1989). Testing for the equivalence of factor covariance and mean structures: The issue of partial measurement invariance. Psychological Bulletin, 105, 456-466. https://doi.org/10.1037/0033-2909.105.3.456

Chen, F. F. (2007). Sensitivity of goodness of fit indexes to lack of measurement invariance. Structural Equation Modeling, 14, 464504. https://doi.org/10.1080/10705510701301834

Cheung, G. W., \& Rensvold, R. B. (2002). Evaluating goodness-of-fit indexes for testing measurement invariance. Structural Equation Modeling, 9, 233-255. https://doi.org/10.1207/S15328007SEM0902_5

Collie, J. R., Shapka, D. J., \& Perry, E. N. (2012). School Climate and Social-Emotional Learning: Predicting Teacher Stress, Job Satisfaction, and Teaching. Efficacy. Journal of Psychology of Education, 104, 1189-1204. https://doi.org/10.1037/a0029356

Davidov, E., Meuleman, B., Cieciuch, J., Schmidt, P., \& Billiet, J. (2014). Measurement Equivalence in Cross-National Research. Annual Review of Sociology, 40, 55-75. https://doi.org/10.1146/annurev-soc-071913-043137

De Simone, S., Cicotto, G., \& Lampis, J. (2016). Occupational stress, job satisfaction and physical health in teachers. European Review of Applied Psychology, 66, 65-77. https://doi.org/10.1016/j.erap.2016.03.002

Hancock, R. G. (2001). Effect size, power, and sample size determination for structured means modeling and mimic approaches to between-groups hypothesis testing of means on a single latent construct. Psychometrika, 66, $373-388$. https://doi.org/10.1007/BF02294440

Helms-Lorenz, M., \& Maulana, R. (2015). Influencing the psychological well-being of beginning teachers across three years of teaching: self-efficacy, stress causes, job tension and job discontent. Educational Psychology, 36, 569-594. https://doi.org/10.1080/01443410.2015.1008403

Honkaniemi, L., Lehtonen, H. M., \& Hasu, M. (2015). Well-being and innovativeness: motivational trigger points for mutual enhancement. European Journal of Training and Development, 39, 393 - 408. https://doi.org/10.1108/EJTD-11-2014-0078

Hu, L., \& Bentler, M. P. (1999). Cutoff criteria for fit indexes in covariance structure analysis: Conventional criteria versus new alternatives. Structural Equation Modeling, 6, 1-55. https://doi.org/10.1080/10705519909540118

Jamal, M. (1999). Job Stress and Employee Well-Being: A Cross-Cultural Empirical Study. Stress Medicine, 15, $153-158$. https://doi.org/10.1002/(SICI)1099-1700(199907)15:3<153::AID-SMI809>3.0.CO;2-0

Kamtsios, S. (2018). Burnout syndrome and stressors in different stages of teachers' professional development: The mediating role of coping strategies. Hellenic Journal of Psychology, 15, 229-25. Retrieved from https://pseve.org/publications/journal/hellenic-journal-of-psychology-volume-15/

Katsantonis, G. I. (2020). Investigation of the Impact of School Climate and Teachers' Self-Efficacy on Job Satisfaction: A CrossCultural Approach. European Journal of Investigation in Health, Psychology and Education, 10(1), 119-133. https://doi.org/10.3390/ejihpe10010011

Katsantonis, G. I. (In-press). Teachers' self-efficacy, perceived administrative support, and positive attitude toward students: Their effect on coping with job-related stress. Hellenic Journal of Psychology, 17(1), 1-14.

Klassen, R., Wilson, E., Siu, A., Hannok, W., Wong, M., Wongsri, N., ... Jansem, A. (2013). Preservice teachers' work stress, selfefficacy, and occupational commitment in four countries. European Journal of Psychology of Education, 28, $1289-1309$. https://doi.org/10.1007/s10212-012-0166-x

Kline, B. R. (2016). Principles and Practice of Structural Equation Modeling (4th ed.). New York, NY, USA: Guilford Press.

Li, H. C. (2016). The performance of ML, DWLS, and ULS estimation with robust corrections in structural equation models with ordinal variables. Psychological. Methods, 21, 369-387. https://doi.org/10.1037/met0000093

Matud, M. P., López-Curbelo, M., \& Fortes, D. (2019). Gender and Psychological Well-Being. International Journal of Environmental Research and Public Health, 16(19), 3531. https://doi.org/10.3390/ijerph16193531

Matud, M. P. (2004). Gender differences in stress and coping styles. Personality and Individual Differences, 37, $1401-1415$. https://doi.org/10.1016/j.paid.2004.01.010

McDonald, P. R. (1999). Test Theory: A Unified Treatment. New York, USA: Lawrence Erlbaum. https://doi.org/10.4324/9781410601087

Mearns, J., \& Cain, E. J. (2003). Relationships between teachers' occupational stress and their burnout and distress: Roles of coping and negative mood regulation expectancies. Anxiety, Stress \& Coping, 16, 71-82. https://doi.org/10.1080/1061580021000057040

Miller, K., Greyling, M., Cooper, C., Lu, L., Sparks, K., \& Spector, E.P. (2000). Occupational stress and gender: a cross-cultural study. Stress Medicine, 16, 271-278. https://doi.org/10.1002/1099-1700(200010)16:5<271::AID-SMI862>3.0.CO;2-G

OECD. (2019). TALIS 2018 Technical Report. Paris, France: OECD Publishing. Retrieved from https://www.oecd.org/education/talis/TALIS_2018_Technical_Report.pdf 
Pecino, V., Mañas, A. M., Díaz-Fúnez, A. P., Aguilar-Parra, M. J., Padilla-Góngora, D., \& López-Liria, R. (2019). Organisational Climate, Role Stress, and Public Employees' Job Satisfaction. International Journal of Environmental Research and Public Health, 16, 1792. https://doi.org/10.3390/ijerph16101792

R Core Team. R: A language and environment for statistical computing. Vienna, Austria: R Foundation for Statistical Computing. Retrieved from https://www.r-project.org/

Rhemtulla, M., Brosseau-Liard, P. É., \& Savalei, V. (2012). When can categorical variables be treated as continuous? A comparison of robust continuous and categorical SEM estimation methods under suboptimal conditions. Psychological Methods, 17, 354373. https://doi.org/10.1037/a0029315

Rosseel, Y. (2012). Lavaan: An R package for structural equation modeling. Journal of Statistical Software, 48, 1-36. https://doi.org/10.18637/jss.v048.i02

Sass, A. D., \& Schmitt, A. T. (2013). Testing Measurement and Structural Invariance: Implications for practice. In Teo, T. (Eds.) Handbook of Quantitative Methods for Educational Research (pp. 315-346). Rotterdam, the Netherlands: Sense Publishers. https://doi.org/10.1007/978-94-6209-404-8_15

Satorra, A., \& Bentler, P. M. (2001). A scaled difference chi-square test statistic for moment structure analysis. Psychometrika, 66, 507-514. https://doi.org/10.1007/BF02296192

Schonfeld, S. I., Bianchi, R., \& Luehring-Jones, P. (2017). Consequences of Job Stress for the Mental Health of Teachers. In M.T. McIntyre, E.S. McIntyre, \& J.D. Francis (Eds.) Educator Stress (pp. 55-76). New York, USA: Springer. https://doi.org/10.1007/9783-319-53053-6_3

Schwarzer, R., \& Hallum, S. (2008). Perceived teacher self-efficacy as a predictor of job stress and burnout: mediation analyses. Applied Psychology: An International Review, 57, 152-171. https://doi.org/10.1111/j.1464-0597.2008.00359.x

Shen, E. Y. (2009). Relationships between self-efficacy, social support, and stress coping strategies in Chinese primary and secondary school teachers. Stress \& Health, 25, 129-138. https://doi.org/10.1002/smi.1229

Skaalvik, M. E., \& Skaalvik, S. (2017). Teacher Stress and Teacher Self-Efficacy: Relations and Consequences. In M.T. McIntyre, E.S. McIntyre, \& J.D. Francis (Eds.) Educator Stress (101-125). New York, USA: Springer. https://doi.org/10.1007/978-3-319-530536_5

Steenkamp, E. M. J.-E., \& Baumgartner, H. (1998). Assessing Measurement Invariance in Cross-National Consumer Research. Journal of Consumer Research, 25, 78-90. https://doi.org/10.1086/209528

Timms, C., Graham, D., \& Caltabiano, M. (2006). Gender Implication of Perceptions of Trustworthiness of School Administration and Teacher Burnout/Job Stress. Australian Journal of Social Issues, 41, 343-358. https://doi.org/10.1002/j.18394655.2006.tb00020.x

Verma, R., Balhara, S. P. Y., \& Gupta, S. C. (2011). Gender differences in stress response: Role of developmental and biological determinants. Industrial Psychiatry Journal, 20(1), 4-10. https://doi.org/10.4103/0972-6748.98407 